\title{
Supercyclic vectors and the Angle Criterion
}

\author{
by \\ Eva A. Gallardo-Gutiérrez (Zaragoza) and \\ JonAthan R. PARTington (Leeds)
}

\begin{abstract}
We show that the Angle Criterion for testing supercyclic vectors depends in an essential way on the geometrical properties of the underlying space. In particular, we exhibit non-supercyclic vectors for the backward shift acting on $c_{0}$ that still satisfy such a criterion. Nevertheless, if $\mathcal{B}$ is a locally uniformly convex Banach space, the Angle Criterion yields an equivalent condition for a vector to be supercyclic. Furthermore, we prove that local uniform convexity cannot be weakened to strict convexity.
\end{abstract}

1. Introduction. Let $\mathcal{B}$ be a complex Banach space. A bounded linear operator $T$ acting on $\mathcal{B}$ is said to be cyclic if there is a vector $x \in \mathcal{B}$ such that the linear span generated by the orbit $\left\{T^{n} x\right\}_{n \geq 0}$ is dense in $\mathcal{B}$. In such a case, the vector $x$ is called cyclic. If the orbit itself is dense, then $T$ is called hypercyclic and $x$ a hypercyclic vector for $T$. While cyclicity is a classical concept, hypercyclic operators have engaged the attention of many operator theorists since the end of the eighties. For a source of references on the subject, updated till 1999, we refer to Grosse-Erdmann's survey [5].

When the scalar multiples of the elements in the orbit are considered, the concept of supercyclic operator arises. We say that a bounded linear operator $T$ acting on $\mathcal{B}$ is supercyclic if there is $x \in \mathcal{B}$, also called supercyclic, such that the projective orbit

$$
\left\{\lambda T^{n} f: n \geq 0 \text { and } \lambda \in \mathbb{C}\right\}
$$

is dense in $\mathcal{B}$. The concept of supercyclic operator was first introduced by Hilden and Wallen [6] in the seventies. Nevertheless, the first example of a supercyclic operator in the Banach space setting can be traced back to Rolewicz's work [10]. He proved that the unilateral backward shift $B$ acting on the sequence space $c_{0}$ or $\ell^{p}, 1 \leq p<\infty$, and defined on the canonical

2000 Mathematics Subject Classification: Primary 47A16, 47A15.

Key words and phrases: supercyclic operators, supercyclic vectors, Angle Criterion.

The first author is partially supported by Plan Nacional I+D grant no. BFM200300034 and Junta de Andalucía FQM-260. 
basis $\left\{e_{n}\right\}_{n \geq 0}$ by

$$
B e_{n}=e_{n-1} \quad(n \geq 1), \quad B e_{0}=0
$$

is supercyclic. Actually, Rolewicz provided concrete examples of supercyclic vectors for $B$ on $c_{0}$ by constructing hypercyclic vectors for complex scalar multiples $\lambda B$ whenever $|\lambda|>1$. For a comprehensive treatment of properties of supercyclic operators, we refer the reader to Montes and Salas's recent survey [9].

Testing whether a vector $x \in \mathcal{B}$ shows any kind of cyclic behaviour for a given operator $T$ seems to be a difficult task. Of course, it is clear that $T^{n} x \neq 0$ for all $n \geq 0$. If, in addition, there exists a non-zero functional $\phi \in \mathcal{B}^{*}$ such that the set of complex numbers $\left\{\phi\left(T^{n} x\right)\right\}_{n \geq 0}$ is not dense in $\mathbb{C}$, then $x$ is not hypercyclic for $T$. This fact was used in [4] to prove that certain operators are not hypercyclic (see the Inner Product Criterion in [4, Chapter 2]). The underlying idea is simple and follows from the fact that the image of a dense set under a non-zero linear functional must be dense. Actually, this idea had been previously used by other authors. For instance, in some applications it can be enough to prove that $\left\{\phi\left(T^{n} x\right)\right\}_{n \geq 0}$ is bounded, or is contained in a straight line, or is the set of powers of a fixed complex number. This latter argument was used in [2, Prop. 4.1] to prove a result of Kitai [7, Cor. 2.4] that asserts that the adjoint of a hypercyclic operator always has empty point spectrum.

On the other hand, in order to check that a given non-zero vector $x$ is not supercyclic for $T$ on a Hilbert space $\mathcal{H}$, it would be enough to find a vector $y$ such that the whole orbit $\left\{T^{n} x\right\}_{n \geq 0}$ lies outside a cone around $y$. It is clear that the scalar multiples of the orbit of $x$ cannot approximate $y$, and consequently, $x$ cannot be supercyclic. This geometric idea was first used in [8, Sec. 5] to construct a non-supercyclic vector for certain weighted shifts on $\ell^{2}$. Moreover, this geometric idea works in any Hilbert space for any operator and provides a Hilbert space criterion for supercyclic vectors, which was given in [3].

The Angle Criterion for Hilbert spaces. Let $T$ be a bounded linear operator on a separable Hilbert space $\mathcal{H}$. Then the vector $x \in \mathcal{H}$ is supercyclic for $T$ if and only if

$$
\sup _{n} \frac{\left|\left\langle T^{n} x, y\right\rangle\right|}{\left\|T^{n} x\right\|\|y\|}=1
$$

for all non-zero vectors $y \in H$.

If we restrict ourselves to the Banach space setting, it follows that a necessary condition for a vector $x \in \mathcal{B}$ to be supercyclic for $T$ is that

$$
\sup _{n} \frac{\left|\phi\left(T^{n} x\right)\right|}{\left\|T^{n} x\right\|\|\phi\|}=1
$$


for all non-zero functionals $\phi \in \mathcal{B}^{*}$. However, as we shall see by means of a counterexample in Section 2, this condition is not sufficient for testing supercyclic vectors in general Banach spaces. Actually, it will depend, essentially, on the geometrical properties of the Banach space.

For this purpose, we recall that a Banach space $\mathcal{B}$ is said to be strictly convex if the conditions $\|x\|=\|y\|=1$ and $\|x+y\|=2$ for vectors $x, y \in \mathcal{B}$ imply that $x=y$. The space $\mathcal{B}$ is locally uniformly convex if for each $x \in \mathcal{B}$ and sequence $\left(y_{n}\right)$ in $\mathcal{B}$ with $\|x\|=1$ and $\left\|y_{n}\right\|=1$ for each $n$, the condition $\left\|x+y_{n}\right\| \rightarrow 2$ implies that $\left\|y_{n}-x\right\| \rightarrow 0$. Clearly local uniform convexity implies strict convexity.

The relevance of these geometric properties to supercyclicity is that, as we shall show in Section 3, the Angle Criterion is necessary and sufficient for supercyclicity if the underlying space is locally uniformly convex, but it is insufficient if the slightly weaker property of strict convexity is assumed.

These convexity conditions are described in many places, for example [1], where it is noted that every separable Banach space can be given an equivalent locally uniformly convex norm. Moreover, the $L^{p}$ spaces with $1<p<\infty$ even have uniformly convex norms, and these are certainly locally uniformly convex. Thus, in principle, the Angle Criterion can be used to test supercyclicity by, if necessary, first passing to an equivalent locally uniformly convex norm.

2. A counterexample in the Banach space setting. In this section, we show that the Angle Criterion is no longer sufficient for testing supercyclic vectors in the Banach space setting.

First, we state the necessary condition for a vector $x$ to be supercyclic in a Banach space $\mathcal{B}$, which is the analogue to that stated for Hilbert spaces.

The Angle Criterion for Banach Spaces. Let $T$ be a bounded linear operator on a separable Banach space $\mathcal{B}$. If the vector $x \in \mathcal{B}$ is supercyclic for $T$ then

$$
\sup _{n} \frac{\left|\phi\left(T^{n} x\right)\right|}{\left\|T^{n} x\right\|\|\phi\|}=1
$$

for all non-zero functionals $\phi \in \mathcal{B}^{*}$.

We include the proof for the sake of completeness.

Proof. Let $\varepsilon>0$ be a positive number. There is a vector $z \in X$ such that $\|z\|=1$ and $|\phi(z)| \geq(1-\varepsilon)\|\phi\|$. Since $x$ is a supercyclic vector, there is a sequence $\left(n_{k}\right)$ and complex numbers $\left(\lambda_{k}\right)$ such that $\left\|\lambda_{k} T^{n_{k}} x-z\right\| \rightarrow 0$. But then

$$
\limsup _{k \rightarrow \infty} \frac{\left|\phi\left(T^{n_{k}} x\right)\right|}{\left\|T^{n_{k}} x\right\|\|\phi\|} \geq 1-\varepsilon
$$

implying (since $\varepsilon$ was arbitrary) that condition (1) holds. 
Recall that $c_{0}$ denotes the Banach space of all sequences $(x(n))_{n \geq 0}$ converging to zero endowed with the supremum norm $\left\|(x(n))_{n \geq 0}\right\|_{\infty}=$ $\max _{n}|x(n)|$. As mentioned in the introduction, Rolewicz [10] showed that if $|\lambda|>1$ then $\lambda B$ is hypercyclic on $c_{0}$ and $\ell^{p}$ for $1 \leq p<\infty$. We have the following

Theorem 2.1. Let $\lambda$ be a real number with $\lambda>1$ and let $T=\lambda B$, regarded as an operator on $c_{0}$. Then $T$ has non-supercyclic vectors satisfying

$$
\sup _{n} \frac{\left|\phi\left(T^{n} x\right)\right|}{\left\|T^{n} x\right\|\|\phi\|}=1
$$

for all non-zero functionals $\phi \in \ell^{1}$.

Proof. We shall construct a vector $x$ for which $\alpha T^{n} x$ is always far from the unit basis vector $e_{0}$, for any scalar $\alpha \in \mathbb{C}$. To do this we fix a real constant $R>\lambda$, and construct an element $x$ of $c_{0}$ which satisfies

$$
\left|\frac{x(n+1)}{x(n)}\right| \geq \frac{1}{R} \quad \text { for each } n,
$$

together with further properties to be specified later. If condition (2) holds, then

$$
\left\|\alpha T^{n} x-e_{0}\right\|_{\infty} \geq \max \left\{\left|\alpha \lambda^{n} x(n)-1\right|,\left|\alpha \lambda^{n} x(n+1)\right|\right\},
$$

but if the first term is less than $1 / 2$, then the second term is at least $1 / 2 R$. Hence such a vector $x$ will not be supercyclic.

To proceed with the construction of $x$, let us fix a dense sequence $\left(\phi_{k}\right)$ in the unit sphere of $c_{0}^{*}=\ell^{1}$ consisting of vectors of finite support of length $L_{k}$. Let $\left(v_{k}\right) \subset c_{0}$ be a sequence of vectors also of finite support of length $L_{k}$ such that $\left|v_{k}(j)\right|=1$ for $0 \leq j<L_{k}$ and $\phi_{k}\left(v_{k}\right)=\left\|\phi_{k}\right\|_{1}=\left\|v_{k}\right\|_{\infty}=1$. Choosing $M_{k}$ sufficiently large such that $R^{L_{k}-M_{k}} \leq \lambda^{-M_{k}}$, we define the norm-one vectors $u_{k}$ as follows:

$$
u_{k}(j)= \begin{cases}v_{k}(j) & \text { if } 0 \leq j<L_{k} \\ R^{L_{k}-j} & \text { if } L_{k} \leq j<M_{k}\end{cases}
$$

Observe that the number of non-zero coordinates of $u_{k}$ is actually $M_{k}$.

Let $\left(N_{k}\right)$ be the sequence of positive integers given by $N_{0}=0$ and $N_{k+1}=N_{k}+M_{k}$. If $S$ denotes the forward shift on $c_{0}$, which satisfies $B S=\mathrm{Id}$, the vector $x \in c_{0}$ we look for is defined by

$$
x=\sum_{k=0}^{\infty} \frac{1}{\lambda^{N_{k}}} S^{N_{k}} u_{k} .
$$

Observe that $x$ satisfies the non-supercyclicity condition (2) since $R^{L_{k}-M_{k}}$ $\leq \lambda^{-M_{k}}$. In addition, by construction, $\left\|T^{N_{k}} x\right\|_{\infty}=1$ as well as $\left|\phi_{k}\left(T^{N_{k}} x\right)\right|$ $=1$. Therefore, 


$$
\sup _{n} \frac{\left|\phi_{k}\left(T^{n} x\right)\right|}{\left\|T^{n} x\right\|\left\|\phi_{k}\right\|}=1
$$

for each $k$. Clearly, the statement of the theorem follows since $\left(\phi_{k}\right)$ is dense in the unit sphere of $c_{0}^{*}$ and this latter fact suffices.

3. Locally uniformly convex Banach spaces. The following result shows that if the underlying Banach space $\mathcal{B}$ is a locally uniformly convex space, then the Angle Criterion in $\mathcal{B}$ is also a sufficient condition for supercyclicity.

THEOREM 3.1. Let $\mathcal{B}$ be a locally uniformly convex Banach space and $T$ a bounded linear operator on $\mathcal{B}$. Then the vector $x$ is supercyclic for $T$ if and only if

$$
\sup _{n} \frac{\left|\phi\left(T^{n} x\right)\right|}{\left\|T^{n} x\right\|\|\phi\|}=1
$$

for all non-zero functionals $\phi \in \mathcal{B}^{*}$.

Proof. It is sufficient to show that the Angle Criterion implies supercyclicity. Let $y \in \mathcal{B}$ be a non-zero vector. We may suppose, without loss of generality, that $\|y\|=1$. By the Hahn-Banach theorem, it is possible to find $\phi \in \mathcal{B}^{*}$ with $\|\phi\|=\phi(y)=1$.

On the other hand, the Angle Criterion implies that there is a sequence $\left(n_{k}\right)$ such that $\phi\left(y_{k}\right) \rightarrow 1$, where $y_{k}=\varepsilon_{k} T^{n_{k}} x /\left\|T^{n_{k}} x\right\|$ and $\left(\varepsilon_{k}\right)$ are complex constants of modulus 1 . Note that $\left\|y_{k}\right\|=1$ for all $k$ and so $\left\|y+y_{k}\right\| \leq 2$.

Since $\left|\phi\left(y+y_{k}\right)\right| \rightarrow 2$ we conclude that $\left\|y+y_{k}\right\| \rightarrow 2$ and by the local uniform convexity we deduce that $\left\|y_{k}-y\right\| \rightarrow 0$. Since this holds for all $y \in \mathcal{B}$, the operator $T$ is supercyclic.

Now let $\mathcal{B}$ denote $c_{0}$ equipped with the equivalent strictly convex norm

$$
\|x\|=\left(\|x\|_{\infty}^{2}+\sum_{n=0}^{\infty} \frac{|x(n)|^{2}}{(n+1)^{2}}\right)^{1 / 2} .
$$

Thus,

$$
\|x\|_{\infty} \leq\|x\| \leq\left(1+\pi^{2} / 6\right)^{1 / 2}\|x\|_{\infty} \quad \text { for all } x \in \mathcal{B} .
$$

We shall use the space $\mathcal{B}$ to show that the Angle Criterion is not sufficient for supercyclicity even in a strictly convex Banach space.

TheOREM 3.2. Let $\lambda$ be a real number with $\lambda>1$ and let $T=\lambda B$, regarded as an operator on the strictly convex space $\mathcal{B}$. Then $T$ has nonsupercyclic vectors such that

$$
\sup _{n} \frac{\left|\phi\left(T^{n} x\right)\right|}{\left\|T^{n} x\right\|\|\phi\|}=1
$$

for all non-zero functionals $\phi \in \mathcal{B}^{*}$. 
Proof. We modify the proof of Theorem 2.1 as follows. Having fixed $R>\lambda$ we can no longer guarantee condition (2), but instead we shall find a vector $x \in \mathcal{B}$ such that for each $n$ there is an $n^{\prime}>n$ with

$$
\left|\frac{x\left(n^{\prime}\right)}{x(n)}\right| \geq \frac{1}{R \beta}
$$

where $\beta$ denotes the constant $\left(1+\pi^{2} / 6\right)^{1 / 2}$ occurring in (4). This guarantees that $\left\|\alpha T^{n} x-e_{0}\right\| \geq 1 / 2 R \beta$ for any scalar $\alpha \in \mathbb{C}$. Therefore, the proof runs similarly to that of Theorem 2.1 .

Again we take functionals $\left(\phi_{k}\right)$ dense in the unit sphere of $\mathcal{B}^{*}$, and we may take them to have finite support of length $L_{k}$. Let $\left(v_{k}\right)$ be also vectors of finite support of length $L_{k}$ such that $\phi_{k}\left(v_{k}\right)=\left\|\phi_{k}\right\|=\left\|v_{k}\right\|_{\infty}=1$. We can no longer take the $v_{k}$ to have coordinates of modulus 1 .

We define the vectors $u_{k}$ by

$$
u_{k}(j)= \begin{cases}v_{k}(j) & \text { if } 0 \leq j<L_{k} \\ 0 & \text { if } L_{k} \leq j<P_{k} \\ \beta^{-1} R^{P_{k}-j} & \text { if } P_{k} \leq j<M_{k} \\ 0 & \text { if } j \geq M_{k}\end{cases}
$$

That is, $u_{k}=v_{k}+w_{k}$, where

$$
w_{k}(j)= \begin{cases}0 & \text { if } 0 \leq j<P_{k} \\ \beta^{-1} R^{P_{k}-j} & \text { if } P_{k} \leq j<M_{k}, \\ 0 & \text { if } j \geq M_{k} .\end{cases}
$$

Here $P_{k}$ is chosen sufficiently large such that $\left\|u_{k}\right\| \leq 1+1 / k$. Observe that we can do this because, first,

$$
\left\|v_{k}\right\|_{\infty} \geq \beta^{-1}
$$

and so, $\left\|v_{k}+w_{k}\right\|_{\infty}=\left\|v_{k}\right\|_{\infty}$. Secondly, we can make the series

$$
\sum_{n=P_{k}}^{\infty} \frac{\left|w_{k}(n)\right|^{2}}{(n+1)^{2}}
$$

arbitrarily small by choosing $P_{k}$ large. Let $M_{k}$ be chosen sufficiently large such that $R^{P_{k}-M_{k}} \leq \beta \lambda^{-M_{k}}$. As in the proof of Theorem 2.1, we consider the sequence $\left(N_{k}\right)$ defined by $N_{0}=0$ and $N_{k+1}=N_{k}+M_{k}$ for each $k \geq 0$. We require that the vector $x$ defined by

$$
x=\sum_{k=0}^{\infty} \frac{1}{\lambda^{N_{k}}} S^{N_{k}} u_{k}
$$

is not supercyclic for $T$.

To check the non-supercyclicity of $x$, observe that since $R^{P_{k}-M_{k}} \leq$ $\beta \lambda^{-M_{k}}$, condition (5) holds for $n=N_{k+1}-1$ and $n^{\prime}$ the index of the numerically largest coordinate of $\lambda^{-N_{k+1}} S^{N_{k+1}} u_{k+1}$, which is at least $\beta^{-1} \lambda^{-N_{k+1}}$ 
in absolute value (the observation that (5) holds for other values of $n$ is immediate from the construction of each $u_{k}$ ). Therefore, $x$ is not a supercyclic vector. However,

$$
\sup _{n} \frac{\left|\phi_{k}\left(T^{n} x\right)\right|}{\left\|T^{n} x\right\|\left\|\phi_{k}\right\|}=1
$$

since $\phi_{k}\left(T^{N_{k}} x\right)=1$ and $\left\|T^{N_{k}} x\right\| \rightarrow 1$ as $k \rightarrow \infty$. Now, the density of $\left(\phi_{k}\right)$ in the unit sphere of $\mathcal{B}^{*}$ yields the statement of the theorem.

Acknowledgements. This work was initiated while the first author was visiting University of Leeds. She is grateful to the Mathematical Department at University of Leeds for its hospitality. The authors are also grateful to the London Mathematical Society for financial support.

\section{References}

[1] Y. Benyamini and J. Lindenstrauss, Geometric Nonlinear Functional Analysis, Vol. 1, Amer. Math. Soc. Colloq. Publ. 48, Amer. Math. Soc., Providence, RI, 2000.

[2] K. C. Chan and J. H. Shapiro, The cyclic behavior of translation operators on Hilbert spaces of entire functions, Indiana Univ. Math. J. 40 (1991), 1421-1449.

[3] E. A. Gallardo-Gutiérrez and A. Montes-Rodríguez, The role of the angle in the supercyclic behavior, J. Funct. Anal. 203 (2003), 27-43.

[4] - - - The role of the spectrum in the cyclic behavior of composition operators, Mem. Amer. Math. Soc. 167 (2004), no. 791.

[5] K. G. Grosse-Erdmann, Universal families and hypercyclic operators, Bull. Amer. Math. Soc. (N.S.) 36 (1999), 345-281.

[6] H. M. Hilden and L. J. Wallen, Some cyclic and non-cyclic vectors of certain operators, Indiana Univ. Math. J. 23 (1974), 557-565.

[7] C. Kitai, Invariant closed sets for linear operators, thesis, Univ. of Toronto, 1982.

[8] A. Montes-Rodríguez and H. N. Salas, Supercyclic subspaces: spectral theory and weighted shifts, Adv. Math. 163 (2001), 74-134.

[9] —, 一, Supercyclic subspaces, Bull. London Math. Soc. 35 (2003), 721-737.

[10] S. Rolewicz, On orbits of elements, Studia Math. 32 (1969), 17-22.

Departamento de Matemáticas

Universidad de Zaragoza

Plaza San Francisco s/n

50009 Zaragoza, Spain

E-mail: eva@unizar.es
School of Mathematics University of Leeds Leeds LS2 9JT, U.K. E-mail: J.R.Partington@leeds.ac.uk

Received April 13, 2004

Revised version September 6, 2004 